\title{
Interview with Henry Hon, CEO and Todd Lane, Marketing Communications Director at Vyew
}

\section{Henry Hon}

is CEO of Vyew, which is a leading and award-winning browser-based visual collaboration and conferencing platform that enables web users to share ideas and create together in real time and over time. Before founding Vyew in 2004, Mr. Hon co-founded five companies: Dahon (1982), the world's largest folding bicycle company; Visionex (1991), a multimedia chip and card manufacturer sold to IBM; Ramax (1995), a chip and SRAM design company whose patents were sold to Intel; ShopD (1998), a B2B import and distribution portal with logistics support sold to a national import distributor; and Authenex (2001), a two-factor authentication and network security company that received more than $\$ 25 \mathrm{~m}$ in venture funding. Mr. Hon has a degree in Industrial Engineering and Operations Research from the University of California, Berkeley.

\section{Todd Lane}

is a high-tech public relations and marketing communications expert whose industry experience includes CAD/CAM, enterprise computing and Web 2.0 collaboration. He has worked both as a consultant in public relations agencies and in-house for high-tech software companies. $\mathrm{He}$ is the author of many feature-length magazine articles, case studies, white papers, news releases and product fact sheets

Keywords: collaboration, social media, social networking, web conferencing, webinar, online meeting, streaming media

Abstract Michael Moon interviews Henry Hon and Todd Lane, of Vyew, a synchronous and asynchronous Web conferencing and visual collaboration platform.

Journal of Digital Asset Management (2007) 3, 317-324. doi:10.1057/palgrave.dam.3650108

MM: Let's start off with your names and titles, and a brief description of the company.

HH: I'm Henry Hon, CEO of the company. The company's name right now is actually Simulāt, but we're using Vyew. So Vyew is the product, and Vyew will actually very soon become the corporate name.

TL: I'm Todd Lane, the marketing communications director at Vyew.

MM: Henry, if you would, give us a corporate history, in terms of where Vyew/Simulät came from - and where you see it headed. HH: Simulāt started as a network simulation provider for large companies for education and training. Our customers included Microsoft, Nortel, McAffee and other large companies with complex network products. We simulated their products and then we used it for their product education and training.

Three years ago we started to build and invest in Vyew. We recognized it as a way of expanding education and training with anything digital. We wanted to make it a space where anything digital can be uploaded, and where people can interact, ask questions and get training. That's the genesis of Vyew.
Since last year, we've dropped building simulations and have completely focused our entire team on developing and extending Vyew. MM: How would you characterize the market category for the Vyew offering?

HH: Vyew is a synchronous and asynchronous webconferencing and visual-collaboration platform.

MM: So by asynchronous, you mean not in real time.

HH: That's correct. That means that it's in a persistent state.

MM: It exists in a storage system, and somebody can get it on-demand.

HH: Exactly. They can access it at any time. Whenever they leave whatever they input or uploaded or interacted with, it will be in that state when someone else comes in.

MM: This gives someone a record of a movie? Or is it just the static state of a previous collaborative session?

HH: You can have a movie. All of the input into our environment is tagged by time, date, user input, name and so on. We have a filter built into the product that lets you filter - again — by timestamp or by name or by project tags or other attributes. 
MM: If I understand you right, there are 2 dimensions - or an overlap of 2 pretty well established categories. There's 1 established market category of Webcast presentations and screen sharing. Right?

HH: Correct.

MM: Then there's another category of online collaboration, which has various attributes or properties to it. One of which is the ability to draw on objects, and add sticky notes or some sort of annotation to particular areas. As well as perhaps some way of recording a particular collaborative session.

HH: That's exactly correct.

It's like a Wiki, a persistent collaborative environment, except that we've also made it highly visual and able to incorporate multimedia.

MM: Another way of saying that is, it's a visual multimedia Wiki.

HH: Exactly. That's very good.

MM: As you find yourself out in the market, you probably get compared and contrasted to a WebEx or an Adobe Connect. Is that correct?

$\mathbf{H H}$ : That is correct on the real-time side. When people think of us as "synchronous," we're definitely compared with WebEx and GoToMeeting. But WebEx and GoToMeeting are not in the asynchronous realm. They're merely synchronous.

When they go to the asynchronous side, there's less. This is more of an emerging space. People are just now finding out that this is a very good way to collaborate when anything digital can be put into a space and collaborated on. People are still finding out the many use cases that the platform can support.

MM: In the asynchronous or recorded ondemand space, whom do you run into there? $\mathbf{H H}$ : Actually, we have not run into that many companies. There are some new Web 2.0 companies that are popping up, like ConceptShare and Octopz. They very much focus on the creative community. We would be compared to them on the asynchronous side. MM: Sure. Also on the asynchronous side, you have some of these on-demand Webcast presentation platforms such as BrainShark or On-24. Maybe even OnStream Media or something like that.

HH: But they're more for Webinar presentations. They don't really have a persistent state, where you allow people to come in and out and upload more content, or make their comments and annotate on content.

MM: Fair enough.

Henry, one of the things that might be useful as a sidebar or footnote here is to share or do a quick overview in terms of things that you've learned about knowledge transfer and knowledge-worker productivity. Lessons learned about those things when you were doing simulations of complex networks.

$\mathbf{H H}$ : When we were doing the simulation of the complex networks it was all in virtual space.

There was no way to really interact with real people. It was all interacting with a program.

We found in that alone there was some drawback, especially when it was anything complex. You need to interact with a real person at a certain point. That's why we began developing Vyew.

In fact, we're finding a number of interactive training companies that are now finding Vyew to be exactly what they want for their training. They have virtual training but at a click they can now interact with real people.

$\mathbf{M M}$ : This gets to a fairly interesting little area in cognitive science that's specifically about how adults learn. One of the findings from a lot of academic and applied research reveals that adults learn best in small peer groups - where there's not an obvious authority figure dictating how things are supposed to be. Where it's more informal and playful.

Specifically, where they come together and share some visual or physical objects.

HH: Absolutely.

MM: Where they communicate, interact and collaborate - using shared objects - to produce some kind of agreed-upon results. HH: You hit it right on the mark, there. To support your point, I'll just give you some of our customers and people that gravitate toward this.

We have a company that does a lot of training work for the military. They are now — to date - our biggest customer. They have interactive training, but the military wanted some real people - groups of people - that can interact together, not just interact with the software.

By embedding their training into our environment, now they have the best of both worlds. They could be trained in a virtual 
environment, but at the same time, they could meet up and group together and give their opinions - and do a project together.

MM: Henry. If I just do a quick footnote on that - essentially, this particular company understands the power of a social network. And more specifically, understands the power of a community of practice.

HH: Right.

MM: Where there are specific ways of getting things done. They want to propagate those practices - if not best practices. The propagation of those best practices requires both a common vocabulary in a persistent state, to your point. And a way by which people can interact with that inventory of objects independent.

But they also come back and - as a group — interact and communicate. They collaborate around the meanings - the context - the actions implied by those particular pieces of content.
HH: Absolutely. You hit it right on.

Again, to this point - The CTO at the Stanford University School of Education, Dr. Paul Kim, has actually joined our team. He's still the CTO there, but he's now part of our team.

The reason is he's been doing a lot of research in exactly what you're saying, in the K-through-20 space, of how to improve learning among that group. One of the findings is exactly that.

It's to have visual objects that students can think through independently, but yet be able to come back together in a group and discuss each others' findings or learnings. A teacher can then jump in at any time and interact independently or with students.

There's one very nice example that this professor is using in one of the elementary schools. He put up some visual objects - I think it was 10 or 15 pictures. This was an English writing class. He said, "Move those objects around to whatever sequence you want

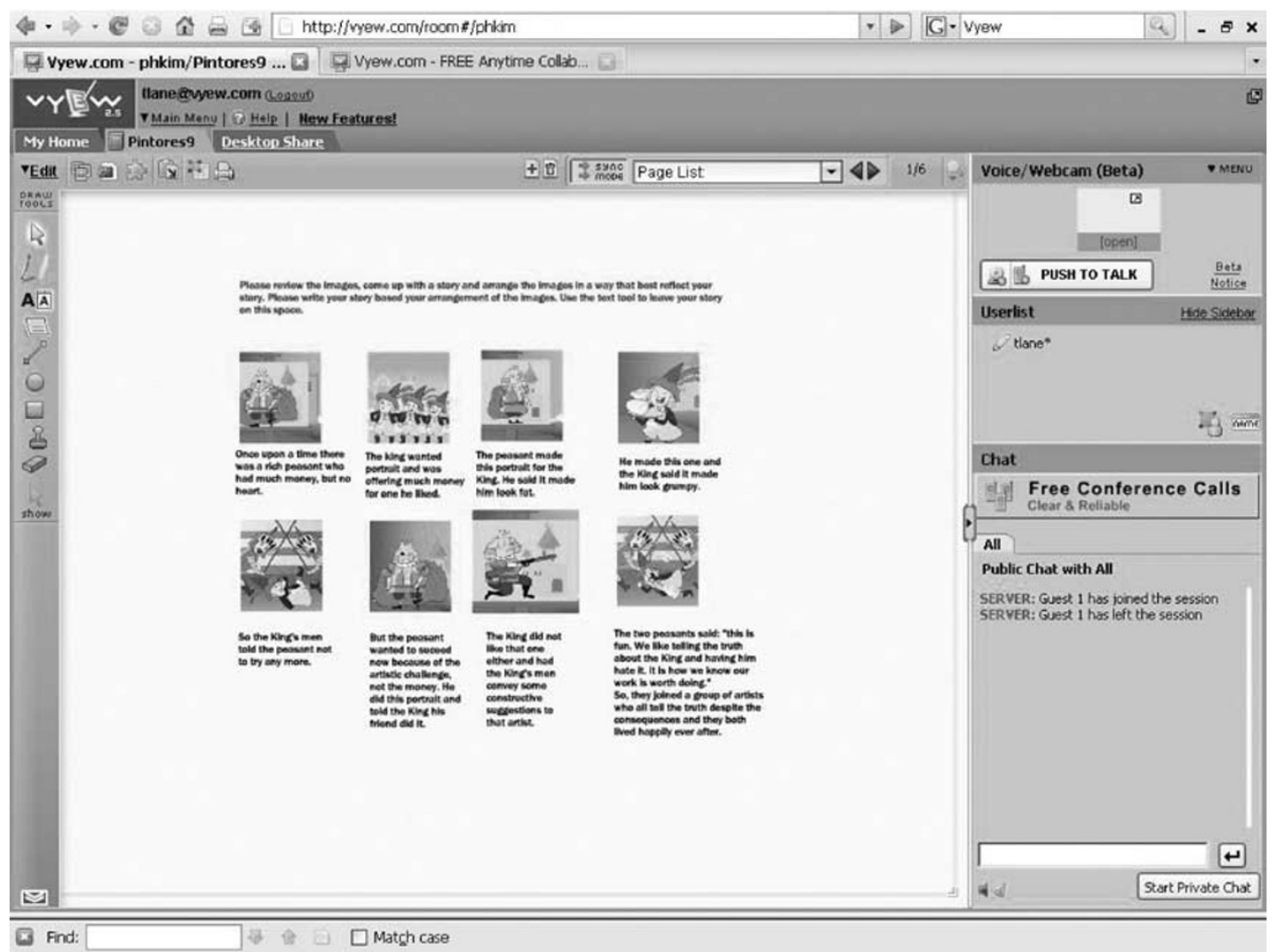

Figure 1: Vyew enables users to share editing tools. A creative writing exercise developed in Vyew by Dr. Paul Kim, CTO of Stanford University School of Education 
and write a caption and story around it."

(Figure 1)

MM: Great.

HH: Now you have 20 or 25 students all coming up with their stories based on these random objects that got thrown in. They all came up with all different stories but they could collaborate together and a teacher could go in and see exactly what they're doing.

MM: That's brilliant.

HH:Yes.

We have another high school teacher who says that through collaboration with Vyew, and this type of learning environment, it's actually changed the social behavior inside a classroom. He noticed that some really shy students who normally would not speak up in class - after going through the environment of this webbased learning - really blossomed. They became active participants, starting from the internet world and now in the physical world.

MM: This gets to a broader social implication, in terms of the challenge of pixilated information. That is to say - again - from cognitive sciences - we've learned that information that's on the screen does not have the same cognitive impact or cognitive effect of reading something intently from a book.

Part of it has to do with the DPI. Part of it has to do with the social contract associated with printed material versus screen material. Specifically, screen materials, you very rarely ever take co-ownership of what's on the screen.

But if I give you a piece of paper - like my business card - it's entirely encouraged for you to write something on the business card that I just gave you. Signifying a transfer of property. HH: Right.

MM: Signifying that this business card - this piece of paper - is now yours, and you are free to do with it as you will. You don't see that in any kind of transfer with electronic "stuff."

However - what we have seen as an alternative way of taking ownership of digital stuff — pixilated material — is through communication, interaction, and collaboration of a group on this side of the scene.

The richest ones, of course, are peer-to-peer, face-to-face interactions. Right?

HH: Right.
MM: For all of the psychological and social reasons. But now what we're beginning to do is to replicate that in a virtual world.

Understanding that the real value is not in the object - nor is it in the presentation. But it's in the communication, the interaction and the collaboration that those objects engender, facilitate and reinforce.

HH: Right. You're making some really excellent points. Normally, people don't feel attached to the digital content. They're looking at it, but they don't feel that they have any ownership. MM: In fact, Henry, one of the tests that I give when I do workshops and seminars and stuff I ask people, "Can you remember a single thing that you read online a month ago? Either an email or a webpage."

Room after room - executive after executive - manager after manager... I get maybe one hand raised in a thousand.

HH: Wow! That's amazing!

MM: It is amazing! When I asked them if they were brought up in the American educational system... I asked them about Andrew Jackson and the Battle of $1812 \ldots$ And I asked them, "What kind of horse is he on?"

60 to 70 percent of the people said, "Oh, he's on a white horse!"

"And is he facing left or right?"

They all say, "left."

Well, it turns out that there's a very famous portrait of Andrew Jackson in the Battle of 1812 down in New Orleans. He's on a white horse, facing west. Right?

HH: Right. I remember that.

MM: But the point is, Henry — we recall things. Again, I'm over 50 years old. I remember things with acuity and precision of things that I read once in high school or junior high school. I'm now recalling a media consumption act of 30 or 40 years ago - where I can't remember a single thing that I read online last week.

The underlying theory of that is that pixilation of information destroys long-term memory.

HH: Interesting.

So how do we correct that? MM:You've already said it. Through communication, collaboration and interaction. HH:Yes. Through communication and through interaction with that pixillated content. 
Here's where the power of the Internet and the computer actually can improve that: by making it both synchronous and asynchronous. Now you have the freedom to interact with content at any time and any place you want because it's in a persistent state. But at the same time, you can just call someone and say, "Let's meet and talk about this."

Now all the parties are looking at the same thing and interacting on the same content. MM: Right.

Again, just to summarize - the value is not what's on the screen, per se. It's in the richness and the depth of the social interaction of what's produced and supported by what's on the screen.

HH: Absolutely. Our job with building Vyew is to make it as easy as possible to upload any type of digital content.

If it's too limited then it starts to break down - and what's the point? We have to be able to bring in any type of office documents and any type of pictures.

If it's MP3 or video or what have you - now you can upload it quickly and allow people to interact with that content in various ways such as with sticky notes or whiteboard or comments. That's the whole idea behind the tool.

MM: Sure. Just take me through another one or two customer success use cases. Put more of a personality and human reality on it.

HH: Maybe Todd can tell us. In fact, we're writing a case study right now. This is a very interesting story.

TL:Yes. We're actively working on case studies with customers. One that's very interesting that I've been working with is called Cellarworks. They specialize in designing architecturally distinguished wine cellars for clients such as celebrities, restaurants and high-end food stores.

For one client - a hotel restaurant - they have to work with many different individuals toward the approval of a design.

For example, they have to work with the restaurant manager and the sommelier - the wine steward - and with other hotel managers on the client side.

Then on the design side, there are designers, architects and a rendering company they work with. There are also HVAC experts - air-conditioning - to make sure that the wine is at the right temperature.

There are also security experts - because the wine is expensive. They have a biometric security system that they implemented.

Then of course there are the contractors who have to build the wine cellar. All of these parties have to work together and they're all from separate organizations.

MM: By the way, Todd - just to punctuate what you've said - all of our research in these various kinds of workflows and so on indicate that review and approval - whether it's conceptual or finished. The review and approval concept remains one of the most difficult, expensive, problem-prone, error-generating activities in most companies.

TL: Yes. You're right.

They were having that same kind of pain with review and approval, coordinating faxes to the various parties, e-mails with attachments or overnight delivery. Then they had to make sure that the changes came back from all the parties and that the changes were incorporated. Then they needed to send the next version to whoever needed to see it.

MM: So Todd, in particular, you're dealing with some media types here that a lot of these conferencing tools aren't really good with. One would be like CAD drawings. Another would be $3 \mathrm{D}$ renders. Another might be navigatable movies. 3D movies. And so on. TL: Yes. (Figure 2)

MM: Take us through some of the unusual media types that this particular cellar designer uses - and of course, their collaboration.

TL: Sure. They have architectural drawings done in CAD software that they have to upload. They also have renderings.

They work with a company in China that does the renderings, so they have a time difference and a language barrier. To communicate visually with this approach really helped the interaction with the Chinese company.

They may even have paper drawings conceptual drawings - that were scanned in. There are also invoices, time sheets and other correspondence. All of these different kinds of files had to be reviewed by various parties at different times. 


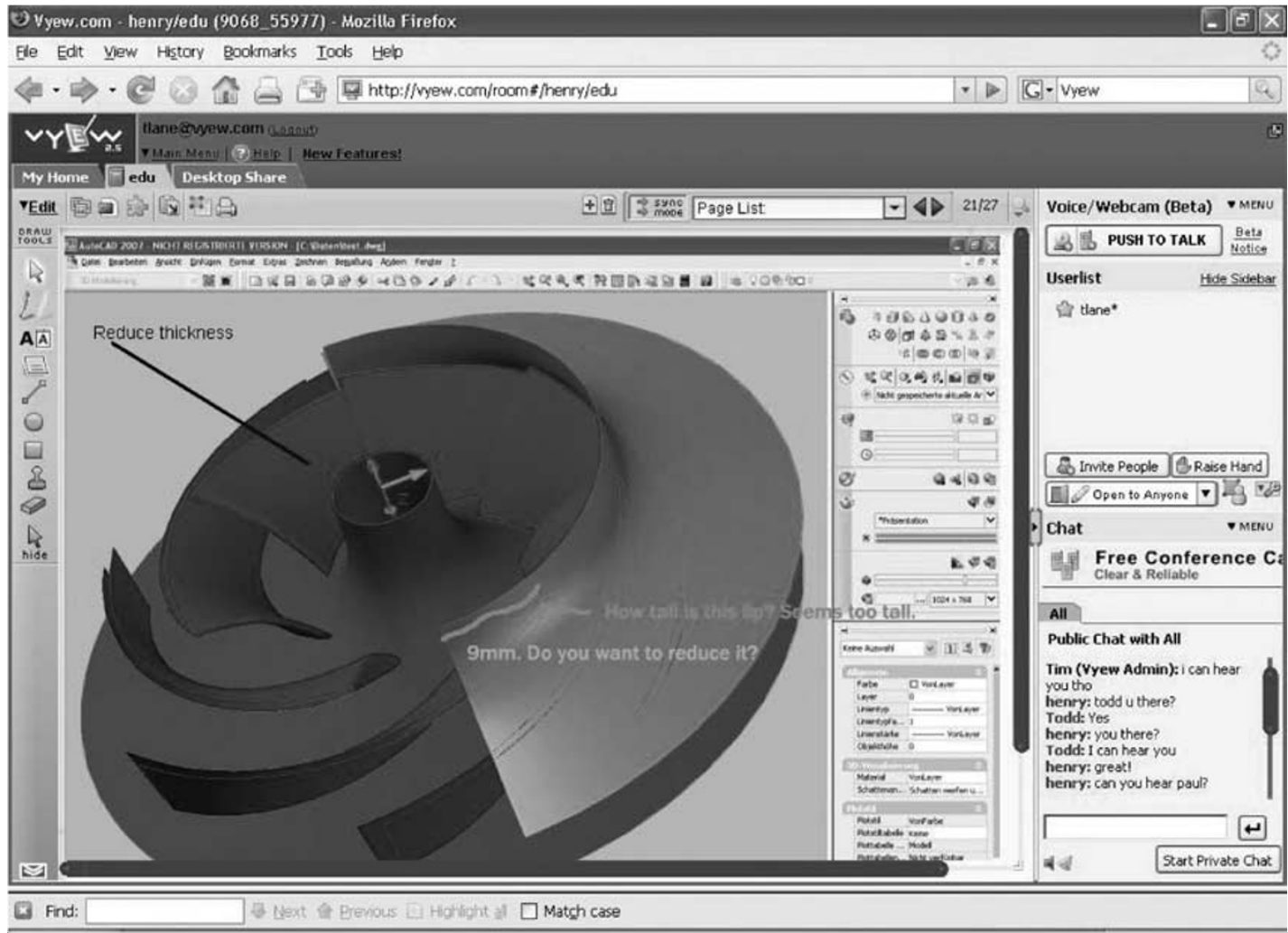

Figure 2: Vyew enables high-level collaboration for CAD drawings and 3D objects. A CAD drawing annotated in Vyew

By switching to Vyew they got rid of all the communication problems that they'd had before - using e-mail, fax, overnight delivery - and then coordinating all the things that came back to make sure they were in the current revision.

With Vyew, they could just put the files in a central repository. Then everyone from anywhere could look at them at their convenience and make annotations. Then they could see who made which annotations, and make sure that they were all incorporated. Then all the parties could view everyone else's annotations, and make sure that they agreed on the final design or the next revision.

They've reported great results from this methodology. Also, their collaborators all love it, because they also don't have to deal with the email, fax or overnight delivery.

HH: Was this the case where they had one project where they couldn't get a final after many days or weeks, and then they used Vyew and it all came together immediately?
TL: That's a different one, a company called Vizwerks. They do retail store and display design. MM: As a category, Todd, they refer to that as "visual merchandising."

TL: They were working with one client, emailing or sending designs back and forth. They went through several revisions over a month and a half. It wasn't supposed to take that long.

When they implemented Vyew, they were able to resolve the issue and get a final design in one call just by being able to collaborate together. They use Vyew regularly with their customers.

MM: So far, we've talked about a couple or 3 areas where Vyew has really made a significant contribution in terms of cycle time, cost and probably quality improvement. Visual merchandising. Architectural design and this learning environment. $\mathrm{K}$ through 20 .

Are there any other major spokes on your hub-and-spoke concept here? Are there any 


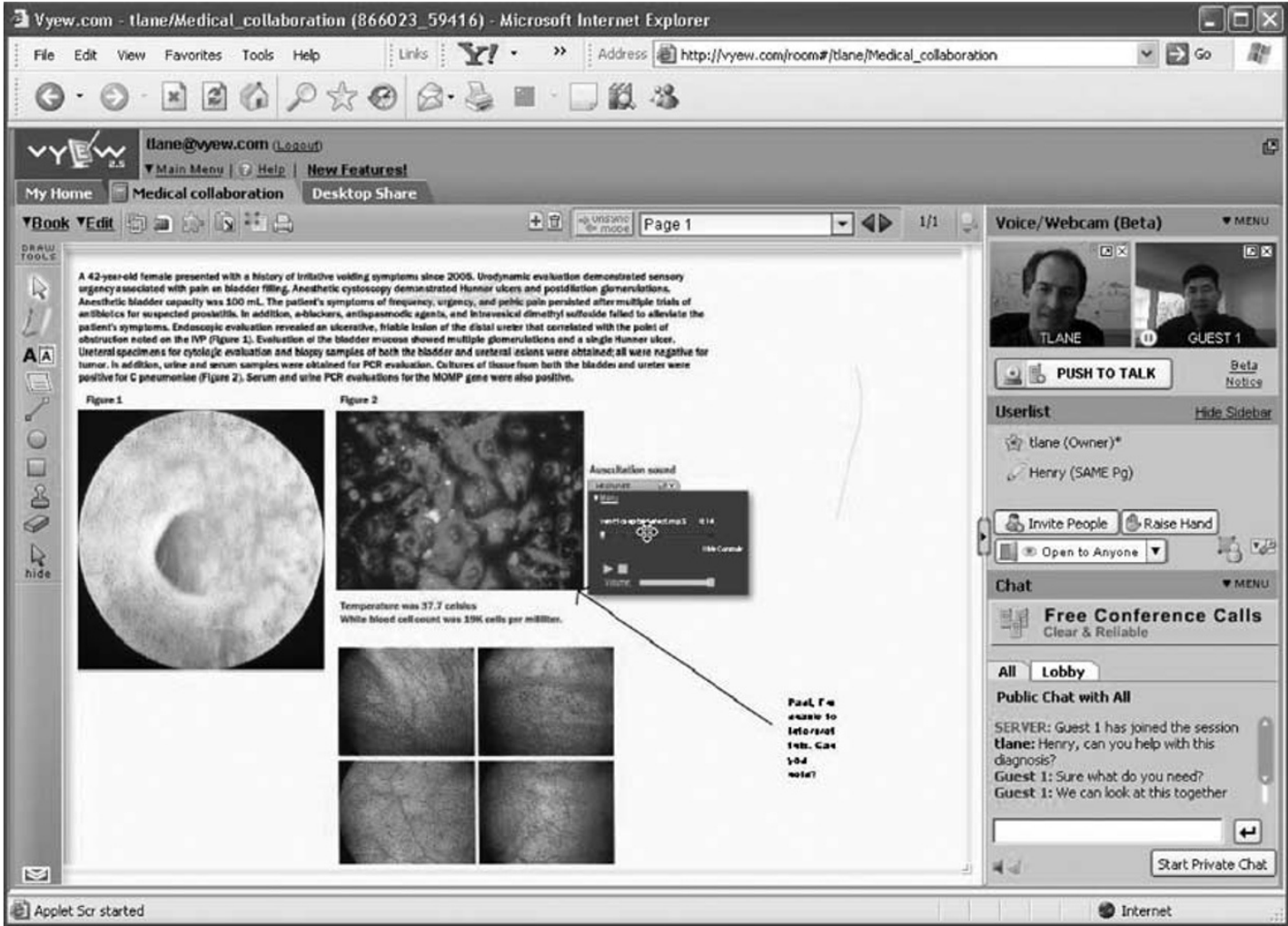

Figure 3: Medical industry-specific example for Vyew collaboration. Collaboration on medical images in Vyew

other spokes to your market, where you see really kind of juicy use cases and good results? HH: Yes. There actually are many.

For example, medical cases. Doctors have to look at X-rays or pictures and show them to other doctors for comment. That is also a big area where collaboration is very helpful (Figure 3). MM: One of the challenges in that medical information - specifically visual medical information - is that oftentimes, these MRI images or X-rays or sonograms or whatever... In many cases, these images are half a gigabyte large. They're huge files.

Oftentimes, the only part that the doctor or medical specialist really wants is just a $1 / 1000$ th slice of it.

HH: Exactly.

MM: How do you facilitate - as opposed to uploading a half a gigabyte file - which is really improbable for everybody except NASA or the DoD - how do you facilitate that? HH: Very simply. We have a one-click screen capture button. So whatever you see on your desktop that you want to share, you just click it and it jumps into Vyew.

MM: In that case, you'd have this large medical image on your display. This X-ray image. Right?

And you'd zoom-zoom-zoom into it. You'd do a screen shot. And that screenshot basically uploads it to Vyew?

HH: Exactly. Now everybody's looking at it either in real time or asynchronously.

MM:You know what would be really cool, Henry, but it's probably not in your product roadmap. It may be in your product roadmap, but probably not in your current thing... To have a dynamic imaging server, so that you could have a little thumbnail in the view, but then you could pan and zoom in it. The contents then being dynamically served from a server. Such as you might find with MediaRich from Equilibrium - or the Scene 7 product - now part of Adobe. Things like that. 
HH: What we'd really like to do is to partner with some of these companies.

MM: Exactly.

HH: We don't want to reinvent the wheel of whatever they're doing but we have our part that could be very useful to them.

We'd love to see ourselves partnering with digital asset management companies that could seamlessly pull images from their system directly into ours.

Your audience is dealing with a lot of digital information and how to manage it. They need a seamless collaboration site. They could pull from their digital library right into Vyew.

MM: Henry, Todd, thank you. 